\title{
Manipulation and Trapping of Particles in the Ray Optics Regime Using DPS-DNG Layered Structures: Toward Calibration and Precision of Optical Tweezers
}

\author{
J. H. Shahbazian \\ Department of ECE, Tufts University, Medford, MA.02155, USA \\ *Corresponding Author: shahbazian.j@gmail.com
}

Copyright (C2014 Horizon Research Publishing All rights reserved.

\begin{abstract}
Optical traps or tweezers use the forces exerted by structured beams of light to confine and manipulate non-intrusively and non-destructively microscopic objects including living cells and bacteria, with high accuracy. This technique provides unique means to control the dynamics of small particles and played a revolutionary role in areas of the physical and biological sciences. This manuscript aims at absolute force calibration of optical beams, using new technique and structure of DPS (Double Positive index of refraction)-DNG (Double Negative index of refraction)layered structure to manipulate targets in the far filed with high resolution trapping scheme. Using this layered structure which acts as a tunable optical band-pass filter would help to calibrate and control the forces on the target(s). The band-pass effect varies with the periodic parameters of the DPS-DNG structure, applied electric and magnetic field, incidence angle and frequency.
\end{abstract}

Keywords Optical tweezer, DNG-DPS, NIM, Optical forces, Gradient force, Optical trapping

\section{Introduction}

Optical tweezers have transformed the ordinary light microscopes from a device for passive observation to a versatile tool for active manipulation and controlled measurement of objects. Optical tweezers are used for trapping particles, assembling objects with the size of micrometer to nanometer. Optical forces provide an expedient way to control the dynamics of small particles in atomic and nonlinear physics, biology and electronics. In spite the fact that the radiation pressure was well known at the beginning of the seventeen century when Johannes Kepler in De Coriolis (1619) published that the radiation pressure causes the tail of comets always point away from the sun no matter where it is located during its journey, but just recently was applied to manipulating particles [1-7]. After that optical tweezers have found interesting applications in manipulating biological cells, such as trapping of viruses and bacterias [8], induced cell fusion [9], studies of chromosome movement [10-12] cellular microscopy [13], and also high definition lithography. The trapping force, which is a gradient force, used in lasers to manipulate targets, could be large enough to accelerate, guide, deflect, and trap targets without inflicting detectable optical damages. This gradient force arises from oscillating electric dipoles that are induced when light passes through objects, which consequently experience a time-averaged force in the direction of the field gradient. When the gradient force overcomes the scattering force, which is the force due to light scattering on the target(s) and acts in the direction of light propagation, trapping is stable. In the optical tweezers the trapping force is described by Hookes law, and force calibrates compare to the trap solidity in the terms of measurable physical parameters of the trap.

Another interesting research picked up in 1968 when Veselago in his paper has shown materials possessing negative electric permittivity and magnetic permeability, simultaneously. These materials exhibits negative index of refraction, anti-parallel group and phase velocities, anti-parallel Poyinting vector and wave vector and form a left handed $\mathbf{k}, \mathbf{E}$, and $\mathbf{H}$ vector set. Because of these properties these materials called left-handed materials (LHM) or double negative index of refraction (DNG) materials. Since then, diverse applications appeared, such as perfect lens and subwavelength focusing $[14,15]$, antennas, couplers and resonators and etc. [16, 17].

In this manuscript a DPS-DNG layered heterostructure used for the focusing of the electromagnetic beams. As 
discussed in $[23,24]$ the DNG layer will act as perfect lens and the DNG-DPS layered structure would able to filter incidence EM waves in an agile and practical way and able to focus on the far filed, subwavelength focal limit. Using this structure would give more degrees of freedom to manipulate the EM beam as shown in [18-20] and the structure could be used in optical tweezing on target particles.

\section{$2 \quad$ Theory and Results}

\section{a- Properties of the structure}

Using the conventional lenses to image objects with applying an appropriate phase and amplitude transformation to the propagating waves, bringing the beams to focus at a certain distance beyond the lens is a simple task. However, according to the Abbes'diffraction limit, using conventional lenses to focus electromagnetic waves and getting the details of an object, smaller than the wavelength is impossible. The reason is, the amplitude of evanescent Fourier components of incidence beam with the transverse wave number of larger than $\frac{2 \pi n}{\lambda}$, which carry information about the sub wavelength details, deteriorates and disappears exponentially. Therefore, the limit of the image resolution of conventional lenses is about the magnitude of the wavelength. Converting the evanescent waves to propagating waves without mixing is the key to extracting the subwavelength information. In references [1315] proposed superlenses that not only would focus the propagating waves, but would also amplify the evanescent waves and enable their detection and both the propagating and evanescent waves would contribute to an image in the far field, resulting in resolution far below the diffraction limit.
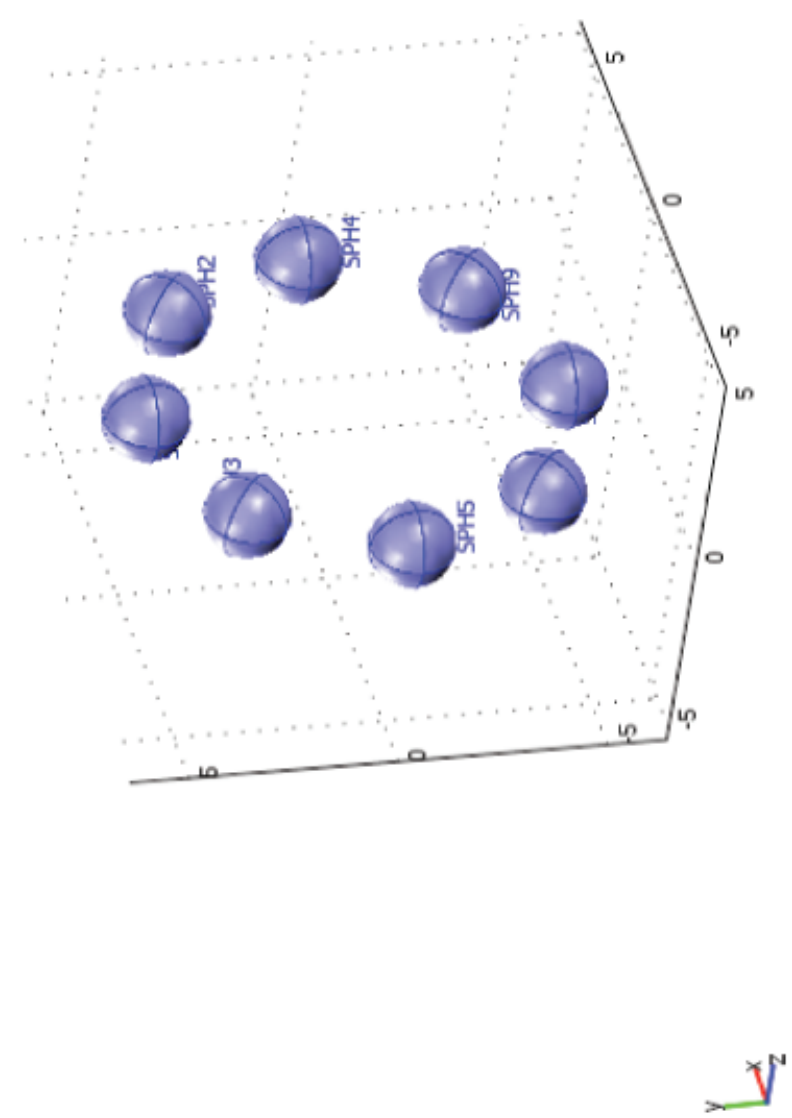

Figure 1. Structure of DNG layer containing 8 or 12 nano spheres on a circular array.

The objective here is to study the manipulation and trap of up to nanometer size dielectric particles with manipulating the electromagnetic waves using layered periodic photonic crystals made with alternating layers of double positive (DPS, ordinary dielectric) materials and double negative materials (DNG). The DPS-DNG test bench would manipulate the refraction of the electromagnetic waves and afterward the force acting on the subwavelength size target. For this manuscript for the DPS layers, germanium has been used and the DNG layer is a test-bed composed of $M g B_{2}$ as host material with twelve or eight polaritonic SiC nanospheres arranged in a circle embedded symmetrically in a ring shown in figure (1). Due to the centrosymmetry of the array there will be no phase difference and would cause coherent enhancement at the focal point.

The DNG layer is anisotropic and the refractive index depends on the direction of the incidence beam. The 
eclectic permittivity, $\varepsilon$, and magnetic permeability, $\mu$, are $3 \times 3$ matrices given by (1);

$$
\varepsilon=\left(\begin{array}{ccc}
\varepsilon_{i i} & 0 & 0 \\
0 & \varepsilon_{j j} & 0 \\
0 & 0 & \varepsilon_{k k}
\end{array}\right) \mu=\left(\begin{array}{ccc}
\mu_{i i} & 0 & 0 \\
0 & \mu_{j j} & 0 \\
0 & 0 & \mu_{k k}
\end{array}\right)
$$

and each element of these matrices are also function of frequency as given by equations (2)and (3) and studied in details in reference [17]. The diameter of the ring is about $6 \mu \mathrm{m}$ and the diameter of each sphere is about $1 \mu \mathrm{m}$, shown in figure (1).

$$
\begin{aligned}
& \varepsilon_{y y}(\omega)=1+\frac{4.8412^{2}}{2.1101^{2}-\omega^{2}} \\
& \mu_{y y}(\omega)=1+\frac{4.2295^{2}}{3.6698^{2}-\omega^{2}}
\end{aligned}
$$

where $\omega=2 \pi f$ and $f$ is frequency in $\mathrm{THz}$.

When a uniform incident plane wave in the Bloch form, $F(x, z+d)=F(0, z) e^{i\left(k_{x} x+K d\right)}$ illuminates the DPSDNG structure, due to difference of the refractive indices between the $\mathrm{SiC}$ nanospheres and the $M g B_{2}$ host medium, the propagating waves will diffract. Here $F(x, z)$ is the incidence field (electric or magnetic depend on the polarization of the incidence field), $k_{x}$ is the wave vector perpendicular to the periodic layers, $K$ is Bloch wave number, $d=d_{1}+d_{2}$ is the thickness of the unit cell and $d_{1}=d f, d_{2}=d(1-f)$ where $\mathrm{f}$ is filling factor. Periodic photonic crystals made with alternating layers of DPS materials and DNG can show unusual photonic band gaps on the top of the ordinary Bragg Band gaps. These gaps called non-Bragg gaps. One kind of non-Bragg band gap arises, when the average refractive index $(\bar{n})$, is zero, the other kinds of non-Bragg band gaps, where either $\mu$ or $\varepsilon$ reaches zero, where the refractive index of the material is zero, and the total reflection at the boundary of a zero refractive index material can be seen, the details shown in reference [17]. Dispersive character of DNG has very important role in non- Bragg band gaps. These reflection gaps (or refraction pass bands) are function of frequency, angle of incidence and depend on the difference between the refractive indices of the layers. Applying static magnetic field, the refractive indices would change and this would cause another degree of freedom to control the bandpass/ forbidden bands of propagating waves as shown in reference [18]. Applying electric field not only would change the band passes but also would change polarization of propagating electromagnetic waves, shown in $[19,20]$. Therefore, using DPS-DNG layered structure as hyper lens would create more degrees of freedom to manipulate the propagating waves.

\section{b- Focal length and point}

As discussed in [13-15, 23-30] and proved by calculation for this manuscript, inside the flat slabs of DNG layer the evanescent waves would exponentially regrow and would be restored to the original amplitude emitted by source and the phase of refracted beams would be corrected and make the subwavelength focusing at the foci possible. In principle, this produces a focusing, in which the fields at foci are an exact replica of the source. The layer of DNG would act as a perfect lens and would manipulate the field and focus the electromagnetic waves to resolutions beyond the diffraction limit in the far field. Figure (2) shows the FTDT simulation of the 3D normalized intensity distribution of the focal spot in the $\mathrm{x}-\mathrm{z}$ transverse cut plane at a distance of about $21 \lambda$ away from the surface of the structure.

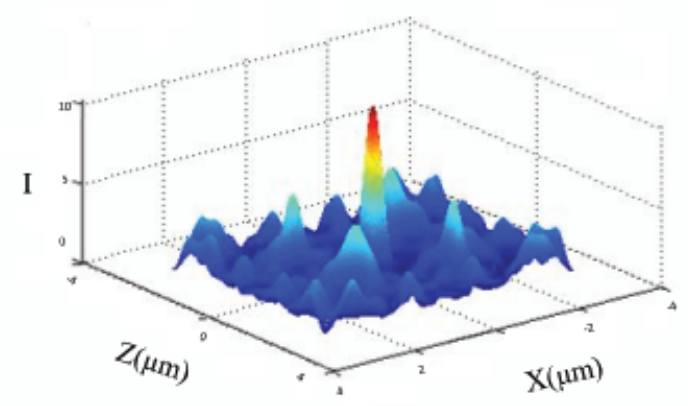

Figure 2. 3-D plot in the $\mathrm{x}-\mathrm{z}$ transverse plane of the focal spot at distance of $21 \lambda$.

In figure (3) the cross section of the focal point and the focusing effecs has been shown. The focal point has elliptical shape with the FWHM about $0.47 \lambda$ along $\mathrm{x}$ axis and $0.53 \lambda$ along $\mathrm{z}$ axis.

In figure (4) the FWHM of the focal spot with normalized intensity distribution at the focus point along the $\mathrm{x}$ axis is shown. In these figures the peak value of the central spot reveals high intensity contrast with the adjacent 


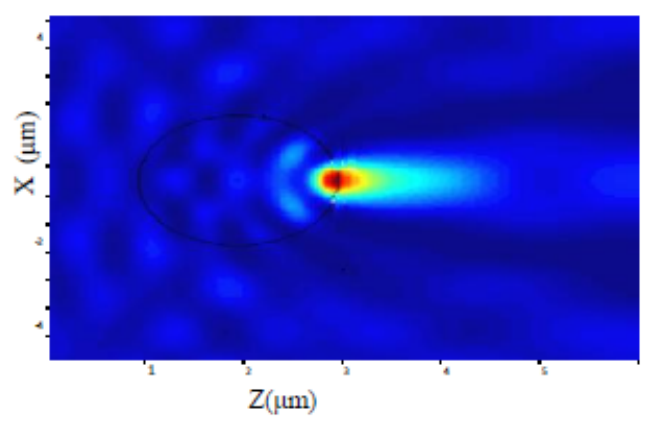

Figure 3. Distribution of intensity alnog the propagation direction.

side lobs. The results demonstrate obviously that the designed structure allows a subwavelength focal spot to be maintained at a larger operating distance and allows a smaller focal spot. Here the size of the focal point depends on the size of the spheres and the circular ring array. With increasing the diameter of the array, the focal length also will increase, but this would cause larger focal spot size. The shorter the focal length, the smaller is the FWHM of the focal spots.

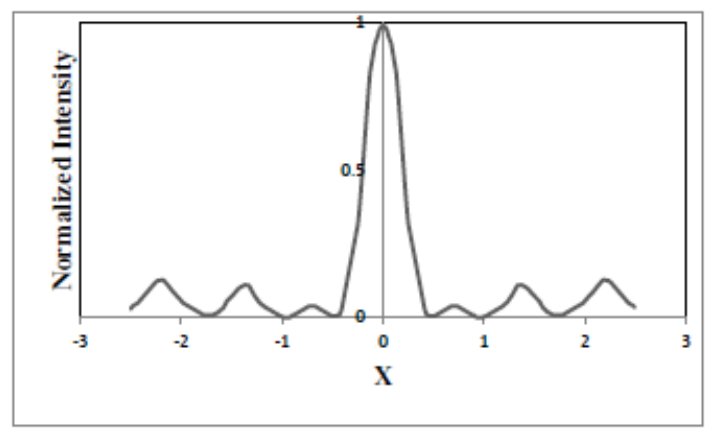

Figure 4. Normalized intensity distribution on $\mathrm{x}$ axis of the focus point.

As known, focusing by a conventional lens, the light intensity distribution of the focal spot depends on the polarization of the incident wavevector. Because the polarization of the wavevector is crucial to the properties of the focus point in the lens system, it has the potential to optimize the focusing effect by choosing proper polarization of the incident wave. But using proposed structure the way shown in reference [20] applying a proper external electric field could change the polarization of the transmitted beam and polarization of the beams should not be a subject of big concern. This is another property of this structure that causes more flexibility and degrees of freedom to manipulate the electromagnetic waves.

The other factors that help manipulation of electromagnetic waves and control the focus of incidence beams are; the refractive indices of the DPS-DNG layers, the incident wavelength and the number of microspheres arranged in the ring. The refractive index of the DNG layer as shown in [17] changes with the frequency, and smaller is this refractive index the output waves will confine tighter and would cause an intense focal point, which is identical to decreasing the wavelength of the incident beam. The focal length shortens, with the increase of the incident wavelength.

\section{c- Optical forces}

Optical tweezers use optical forces which are sensitive to small perturbations, to trap targets. One kind of force called the gradient force which arises from oscillating electric dipoles and is induced when electromagnetic waves transmit in objects in the direction of the field gradient. Another force called scattering force and is due to the light scattering by targets in the direction of light propagation. When the gradient force overcomes the scattering force, the radiation pressure creates a stable, three-dimensional equilibrium and the trapping is being stable. If the target size $d>>\lambda$ the regime called geometrical optics regime or Mie regime, and one can use the ray-optics to explain the phenomenon. In this regime the electromagnetic beams carry momentum and bent while passing through a target (dielectric sphere) with a refractive index $n$, greater than the surrounding medium. Using conservation of momentum, the rate of change of momentum in the deflected rays, bears an equal and opposite rate of change in momentum to the target particle. When a dielectric sphere is placed in a light gradient, the sum of all beams transmitting through it generate gradient in force, and push the sphere towards the more intense region of the beam. In this regime the incident rays reflect and refract at the surface of the target and give rise to optical forces. The force, $\mathrm{F}$, due to incident ray with the power, $\mathrm{P}$, is given by; 
$F=\frac{n_{m} P}{c}\left\{1+R \cos 2 \theta_{i}-\frac{T^{2}\left[\sin \left(2 \theta_{i}-2 \theta_{r}\right)+R \cos 2 \theta\right]}{1+R^{2}+2 R \cos 2 \theta_{r}} \hat{k}\right\}+\frac{n_{m} P}{c}\left\{R \sin 2 \theta_{i}-\frac{T^{2}\left[\sin \left(2 \theta_{i}-2 \theta_{r}\right)+R \sin 2 \theta\right]}{1+R^{2}+2 R \cos 2 \theta_{r}} \hat{i}\right\}$

where $\theta_{i}$ is the incidence angle, $\theta_{r}$ is the angle of refraction, $i$ and $k$ are unit vectors along $\mathrm{x}$ and $\mathrm{z}$ direction, $\mathrm{R}$ and $\mathrm{T}$ are the reflection and refraction coefficients, $n_{m}$ is the index of refraction of the surrounding medium and $\mathrm{c}$ is the speed of light. The first part of the equation is scattering force and the second part is the gradient force and $\mathrm{F}$, the total force exerted by a incidence beam is the vector sum of all the scattering and gradient forces.

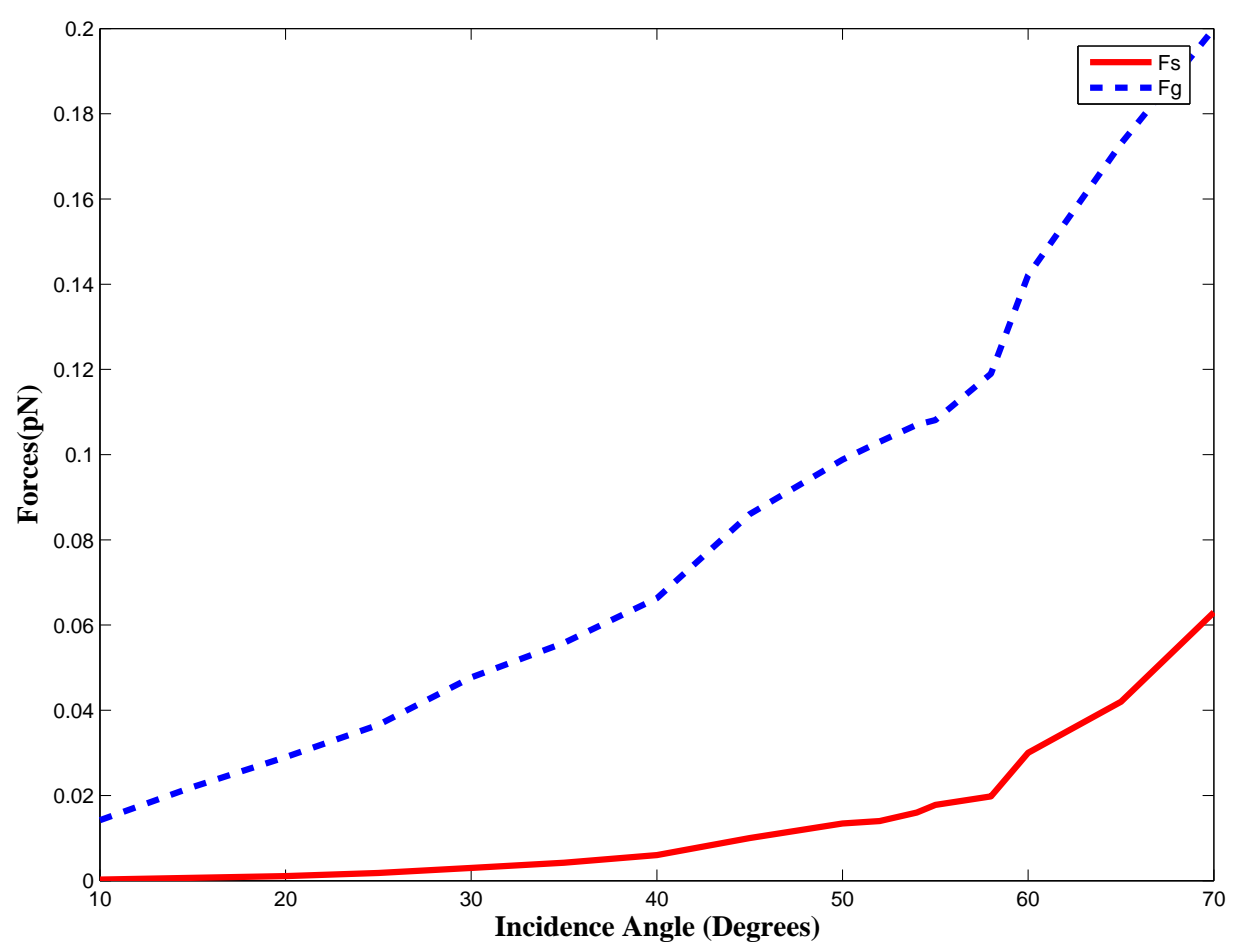

Figure 5. Gradient and scattering forces vs. incidence angle.

In figure (5), the gradient $F_{g}$ (dashed, blue line)and scattering force $F_{s}$ (solid, red line) incidence incident angle $\theta_{i}$ are plotted. As can be seen from the graph, the scattering force is zero or close to zero for small incidence angles (up to ten degree), while the gradient force is much larger than the scattering force start form the small incidence angle and growing in magnitude for higher angles of incidence. Increasing the incidence angle the $F_{g}$ increases faster than the scattering force, giving the vertical displacement of the particle towards higher intensity of the beam and the optical axis. This will cause more stable equilibrium and trapping of targets.

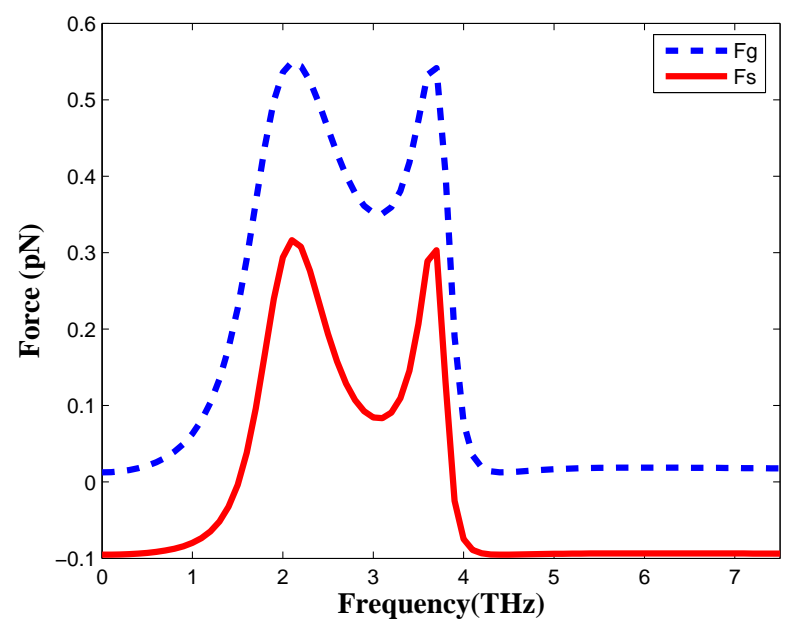

Figure 6. Gradient and scattering forces vs. incidence frequency of the incidence beam.

In figure (6) the $F_{g}$ and $F_{s}$ vs frequency of the incidence wave is shown. The $F_{g}$ stays always positive (blue 
dashed line) for all the incidence frequencies but the $F_{s}$ for small frequencies of below 150 and bigger than 400 $\mathrm{GHz}$ has negative value. This means the particle will not have a point of stable equilibrium in the same horizontal plane of the focus; rather, this position will be below the optical axis.

\section{Conclusion}

The DPS-DNG layered structure would filct like band pass/ band gap filter for transmitted waves. One kind of band which called Bragg band gap is in the base of interference of incidence waves and the other kind called non Bragg band gaps These gaps forbid transmission of electromagnetic waves with certain frequencies. These bands can be manipulated by several factors like periodicity and the size of the structure, frequency of the incidence waves, difference between the refractive indices of the layers. The incidence angle, applied electric and magnetic fields also change these band gaps, stronger the external fields, wider the band gaps become. Applying electric field with proper strength would change the polarization of the transmitted beams.

Using DPS-DNG layers to tweeze and focus electromagnetic waves on targets is a new and efficient way. With using this structure which will act as a superlens one can manipulate and focus electromagnetic waves to resolutions beyond the diffraction limit. This would allow a subwavelength focal spot to be maintained at a larger operating distance, or alternatively allows a smaller focal spot than could be achieved using a conventional lens at a fixed distance.

Using this layered structure in optical tweezing will result more degrees of freedom to target particles with different size, bonding force and sensitivity in the case of cells and biological targets. The size of focal spot may be reduced at the expense of the spot intensity and the emergence of the sidebands which contain the vast majority of the optical intensity.

\section{REFERENCES}

[1] A. Ashkin, Acceleration and trapping of particles by radiation pressure, Phys. Rev. Lett. 24, 156159 (1970).

[2] ARTHUR ASHKIN , Optical trapping and manipulation of neutral particles using lasers,Proc. Natl. Acad. Sci. USA, Vol. 94, pp. 48534860, May 1997

[3] A. Ashkin, Applications of laser radiation pressure, Science 210, 10811088 (1980).

[4] A. Ashkin, J. M. Dziedzic, J. E. Bjorkholm, and S. Chu, Observation of a single-beam gradient force optical trap for dielectric particles, Opt. Lett. 11, 288290 (1986).

[5] P. A. Maia Neto and H. M. Nussenzveig, Theory of optical tweezers, Europhys. Lett. 50, 702708 (2000).

[6] Y. Roichman, B. Sun, Y. Roichman, J. Amato-Grill, and D. G. Grier, Optical forces arising from phase gradients, Phys. Rev. Lett. 100, 013602 (2008).

[7] A. Mazolli, P. A. Maia Neto, and H. M. Nussenzveig, Theory of trapping forces in optical tweezers, Proc. Royal Soc. London A 459, 30213041 (2003).

[8] Ashkin, A. and J.M. Dziedzic, Optical trapping and manipulation of viruses and bacteria. Science, 1987. 235(4795): p. $1517-20$.

[9] R. W. Steubing, S. Cheng, W. H. Wright, Y. Numajiri, and M. W. Berns, Laser induced cell fusion in combination with optical tweezers: the laser cell fusion trap, Cytometry 12, 505-510 (1991).

[10] M. W. Berns, W. H. Wright, B. J. Tromberg, G. A. Profeta, J. J. Andrews, and R. J. Walter, Use of a laserinduced optical force trap to study chromosome movement on the mitotic spindle, Proc. Natl. Acad. Sci.U.S.A. 86, 7914-7918 (1989).

[11] V. Emiliani et al., Wave front engineering for microscopy of living cells, Opt. Express 13, 1395-1405 (2005).

[12] V.G. Veselago, "The electrodunamics of substances with simultaneously negative values of $\epsilon$ and $\mu$," Sov. Phys. Usp. 10,509-514 (1968).

[13] J. B. Pendry, Negative Refraction Makes a Perfect Lens,Phys. Rev. Lett. 85, 39663969 (2000).

[14] Jinho Lee, Kwangchil Lee, Haesung Park, Gumin Kang, Dai-Hyuk Yu, and Kyoungsik Kim, Tunable subwavelength focusing with dispersion-engineered metamaterials in the terahertz regime, Optics Letters, Vol. 35, Issue 13, pp. 22542256 (2010)

[15] Ziolkowski, R.W.; Kipple, A.D., Application of double negative materials to increase the power radiated by electrically small antennas, IEEE Transactions on Antennas and Propagation, Volume: 51 Issue:10 p: 2626 2640(2003).

[16] Wang, Rui; Yuan, Bo; Wang, Gaofeng; Yi, Fan "Efficient Design of Directive Patch Antennas in Mobile Communications Using Metamaterials". International Journal of Infrared and Millimeter Waves Volume 28, Issue 8, pp.639-649,(2007). 
[17] J.Shahbazian, A.Karakashian, "Reflection of visible light on the Anisotropic Slab of DPS-DNG Layers", JOSA B Vol. 26, B1-B6 (2009).

[18] J.H.Shahbazian, A.S.Karakashian, "Magneto-optical effect on DPS-DNG layered structure", JOSA B, Vol. 26, Issue 12, 2342-2346 (2009).

[19] J.H.Shahbazian, A.S.Karakashian, "Linear electro -optical effect on DPS-DNG layered structure", IJMP, Vol 23,No. 15, 3205-3211 (2009).

[20] J. H. Shahbazian, Electro-optical Modulators using DPS-DNG Layered Structure, ISRN Optics, vol. 2012, Article ID 957189, (2012).

[21] C. , Luo, S. G. , Johnson, J. D. ,Joannopoulos, J. B. Pendry, All-angle negative refraction without negative effective index, Phys. Rev. B 65201104 (2002).

[22] S., Foteinopoulou, E. N., Economou, C. M., Soukoulis, Refraction in Media with a Negative Refractive Index, Phys. Rev. Lett. 90 107402, (2003).

[23] Zhifang Feng, Xiangdong Zhang, Kun Ren, Shuai Feng, Zhi-Yuan Li, Bing-Ying Cheng, Dao-Zhong Zhang, Experimental demonstration of non-near-field image governed by negative refraction law, Phys. Rev. B 73075118 (2006).

[24] S., Foteinopoulou, C. M., Soukoulis, Negative refraction and left-handed behavior in two-dimensional photonic crystals, Phys. Rev. B 67235107 (2003).

[25] Parimi P V, Lu W T, Vodo v, Sokoloff J, Derov J S and Sridhar S, Negative Refraction and Left-Handed Electromagnetism in Microwave Photonic Crystals, Phys. Rev. Lett. 92 127401(2004).

[26] E., Cubukcu, K., Aydin., E, Ozbay, S., Foteinopoulou, C. M., Soukoulis, Subwavelength Resolution in a TwoDimensional Photonic-Crystal-Based Superlens,Phys. Rev. Lett. 91 207401(2003).

[27] K., Guven, K., Aydin, K.B., Alic, C.M.,Soukoulis, E., Ozbay, Spectral negative refraction and focusing analysis of a two-dimensional left-handed photonic crystal lens, Phys. Rev. B 70 205125(2004).

[28] C., Luo, S. G., Johnson, J.D., Joannopoulos, J.B., Pendry,Unrestricted superlensing in a triangular twodimensional photonic crystal, Phys. Rev. B 68045115 (2003).

[29] X., Wang, Z. F., Ren,K., Kempa, Improved superlensing in two-dimensional photonic crystals with a basis, Appl. Phys. Lett. 86061105 (2005).

[30] R. Moussa, S., Foteinopoulou, L., Zhang, G., Tuttle, K., Guven, E., Ozbay, C. M., Soukoulis, Negative refraction and superlens behavior in a two-dimensional photonic crystal, Phys. Rev. B 71085106 (2005). 